\title{
Insulin Signaling in Bone Marrow Adipocytes
}

\author{
Michaela Tencerova ${ }^{1,2} \cdot$ Meshail Okla ${ }^{3,4} \cdot$ Moustapha Kassem ${ }^{1,4,5}$
}

Published online: 20November 2019

(C) The Author(s) 2019

\begin{abstract}
Purpose of Review The goal of this review is to discuss the role of insulin signaling in bone marrow adipocyte formation, metabolic function, and its contribution to cellular senescence in relation to metabolic bone diseases.

Recent Findings Insulin signaling is an evolutionally conserved signaling pathway that plays a critical role in the regulation of metabolism and longevity. Bone is an insulin-responsive organ that plays a role in whole body energy metabolism. Metabolic disturbances associated with obesity and type 2 diabetes increase a risk of fragility fractures along with increased bone marrow adiposity. In obesity, there is impaired insulin signaling in peripheral tissues leading to insulin resistance. However, insulin signaling is maintained in bone marrow microenvironment leading to hypermetabolic state of bone marrow stromal (skeletal) stem cells associated with accelerated senescence and accumulation of bone marrow adipocytes in obesity.

Summary This review summarizes current findings on insulin signaling in bone marrow adipocytes and bone marrow stromal (skeletal) stem cells and its importance for bone and fat metabolism. Moreover, it points out to the existence of differences between bone marrow and peripheral fat metabolism which may be relevant for developing therapeutic strategies for treatment of metabolic bone diseases.
\end{abstract}

Keywords Bone marrow adipose tissue $\cdot$ Marrow adiposity $\cdot$ Insulin signaling $\cdot$ Bone marrow mesenchymal stem cells

\section{Introduction}

Bone marrow adipose tissue (BMAT) comprises approximately $8 \%$ of total fat mass and thus representing a significant fat

This article is part of the Topical Collection on Bone Marrow and Adipose Tissue

Michaela Tencerova

mtencerova@health.sdu.dk

1 Department of Molecular Endocrinology, KMEB, University of Southern Denmark and Odense University Hospital, 5000 Odense C, Denmark

2 Present address: Department of Molecular Physiology of Bone, Institute of Physiology, Czech Academy of Sciences, 14220 Prague 4, Czech Republic

3 Department of Community Health Sciences, College of Applied Medical Sciences, King Saud University, Riyadh, Saudi Arabia

4 Stem Cell Unit, Department of Anatomy, College of Medicine, King Saud University, Riyadh, Saudi Arabia

5 Department of Cellular and Molecular Medicine, The Novo Nordisk Foundation Center for Stem Cell Biology (DanStem), Panum Institute, University of Copenhagen, Copenhagen, Denmark depot in adult humans with a role in bone homeostasis and whole body energy metabolism [1]. BMAT is more predominant in the appendicular than in axial skeleton [2]. The fatty acid composition of BMAT varies considerably based on its anatomical location, but tibia BMAT was found to have a fatty acid profile that resembles white and classical brown adipose tissues [3]. In the post-natal organism, BMAT originates from progenitor cells that are distinct from peripheral adipose tissues. BMAT is thought to be derived from bone marrow stromal (skeletal, mesenchymal) stem cells (BMSC) present within the bone marrow stroma and that are capable for differentiation, in addition to adipocytes, into osteoblasts and chondrocytes [1]. Currently, there is no consensus regarding the phenotype of BMAT progenitors but a number of markers have been proposed to identify adipocyte progenitors within the bone marrow, including osterix [4, 5], Prx1 and Nestin1 (reviewed in [6]), leptin receptor [7], Rankl [8], Znf423 [9••], and Hoxa11 [10].

BMAT plays a role in lipid storage, skeletal remodeling, and hematopoietic regulation but the mechanisms mediating and integrating these diverse functions have not yet been fully delineated [3]. In addition, BMAT is recognized as an endocrine organ producing local and systemic factors including 
adiponectin, dipeptidyl peptidase 4 (DPP4), legumain (LGMN), secreted frizzled-related protein 1 (sFRP-1), and delta-like 1 (DLK1) (also known as preadipocyte factor 1 (Pref1)) $[9 \bullet \cdot, 11 \bullet, 12-14]$. BMAT undergoes pathologic changes during aging and in a number of diseases $[1,2,4]$; e.g., it expands in anorexia nervosa, states of estrogen deficiency, glucocorticoid excess, and growth hormone deficiency $[2,11 \bullet \cdot]$.

Another evidence about the role of BMAT to participate in regulating whole body energy metabolism is its ability to respond to insulin [15], to activate Sirt1, a key cellular energy sensor, and to induce a thermogenic gene program [16]. BMAT may contribute to systemic glucose and fatty acid clearance [3]. In addition, BMAT responds to insulinsensitizing anti-diabetic medications such as thiazolidinedione (TZD) drugs, PPAR $\gamma$ agonists $[4,15,17]$.

In the current review, we will discuss the role of insulin signaling in bone marrow adipocyte formation, metabolic functions, and its contribution to cellular senescence (Fig. 1). We will also summarize common factors involved in the regulation of BMSC differentiation fate into BMAT with a special reference to obesity and type 2 diabetes (T2D).

\section{Insulin Signaling in Bone Marrow Adipocytes}

\section{Insulin and Insulin Receptor}

Bone marrow adipocytes express insulin receptors $[18 \bullet \bullet, 19]$ and insulin signaling is essential for BMAT formation and function (reviewed in [17]). Ablation of insulin receptor decreases BMAT volume in distal tibia as a result of a reduction in adipocyte size, but not in number [20]. In genetically reconstituted insulin receptor knockout mice (that are euglycemic as a result of human insulin receptor transgene expression in the pancreas, liver, and brain), BMAT is suppressed and whole tibial mRNA level of aP2, a marker of mature adipocytes, is also reduced compared with wild-type tibias [21]. On the other hand, insulin signaling activation in HFD-fed mice associates with elevations in adipogenic genes and expansion in BMAT [18••]. Interestingly, BMAT expansion accompanying obesity has no adverse effects on insulin sensitivity in marrow adipocytes, unlike peripheral white adipocytes, which manifested impaired insulin sensitivity [18••]. Similar to obese mice, activation of insulin signaling in obese humans stimulates a pro-adipogenic differentiation of BMSC [18••, 22••] (depicted in Fig. 1). Adults with morbid obesity and $\mathrm{T} 2 \mathrm{D}$, who have high serum insulin levels, exhibited higher total BMAT at the lumbar spine and femoral metaphysis compared with those without diabetes [23]. Thus, current literature suggests that alterations in insulin receptor function or insulin level regulate BMAT formation.

\section{Insulin Receptor Substances 1 and 2}

Insulin responses are mediated largely through signaling substrates insulin receptor substance 1 (IRS-1) and insulin receptor substance 2 (IRS-2) [24] and a number of studies have demonstrated that IRS- 1 and IRS-2 play a regulatory role in BMAT formation. IRS-1 and IRS-2 are expressed in BMAT and regardless of BMAT expansion, their gene expression was not reduced by HFD in obese mice as observed in visceral fat [18・•]. In fact, IRS-1 gene expression was induced by prolonged HFD (20 weeks) [18••]. The specific role of IRS-

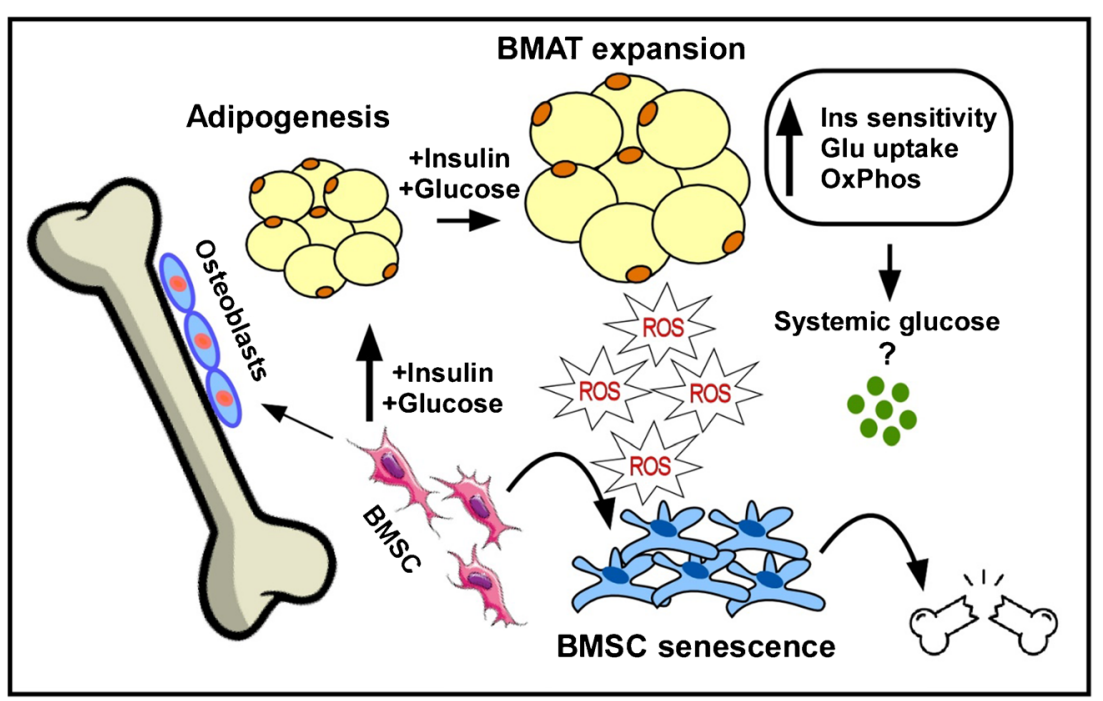

Fig. 1 A working model for the role of insulin signaling in bone marrow adipocyte development, metabolic function and cellular senescence. Active insulin signaling, as in the state of obesity, increases the proadipogenic potential of bone marrow stromal stem cells (BMSC) leading to bone marrow adipose tissue (BMAT) expansion, which is associated with enhanced insulin sensitivity, glucose uptake, and oxidative phosphorylation. This metabolic phenotype of BMSC in obesity results in increased ROS production, which might lead to creation of senescent bone marrow microenvironment and stem cell exhaustion contributing to bone fragility in metabolic bone diseases 
1 and IRS-2 in BMAT formation in vivo has not been studied. A recent in vitro study, however, showed that IRS-1 negatively regulates rodent BMSC adipogenesis as overexpression of IRS-1 decreased the gene expression of adipogenic markers Cebp $\beta$ and Ppar $\gamma$, while IRS-1 deficiency upregulated Cebp $\beta$ and Ppar $\gamma$. The mechanism by which IRS-1 regulates rat BMSC adipogenesis in this study was mediated partially by PI3K-AKT and MEK-ERK pathways [25]. Contrary to IRS-1, IRS-2 positively regulates adipogenesis in human BMSC as Irs-2 expression was induced during adipogenesis, while IRS2 deficiency repressed adipogenesis and led to downregulation of Cebp $\alpha$ and Ppar $\gamma$. In addition, targeting IRS-2 in human BMSC by miR-431 negatively regulated adipogenesis and decreased the gene expression of Cebp $\alpha$ and Ppar $\gamma$ [24]. IRS-2 can compensate for IRS-1 dysfunctions as BMSC of IRS-1-deficient mice showed induced IRS-2 expression, which was reported to be mediated by miR-33 downregulation [26].

\section{Insulin-Like Growth Factor-1}

Insulin-like growth factor-1 (IGF-1) plays an important role in the regulation of bone marrow adiposity [24, 27]. IGF-1 mediates biological effects mostly through binding to IGF-1 receptor and with less affinity to insulin receptor [28, 29]. There is a negative correlation between plasma IGF-1 and BMAT formation [30]. In subjects with a severely reduced caloric intake, dysregulation of the growth hormone-IGF-1 axis and low leptin levels are associated with increased BMAT formation [31]. In obese women who underwent bariatric surgery, the increase in serum IGF-1 levels is associated with declines in BMAT volume. In addition, in a cross-sectional study, obese premenopausal women with higher levels of IGF-1 have lower vertebral marrow fat content independent of age and BMI [32]. Likewise, growth hormone replacement in rats that underwent hypophysectomy exhibit reduced BMAT [33]. Moreover, BMSC obtained from mice with decreased serum IGF-1, due to knockdown of IGF-1 production by the liver or knockout of its binding proteins, revealed a greater adipogenic potential compared with controls [34]. In vivo analysis of the bone marrow of IGF-1 mutant mice showed elevated levels of adipogenic markers [34].

\section{IGF-Binding Protein 4}

IGF-binding proteins (IGFBPs) are regulators of tissue levels and functions of IGF. Among IGFBPs, IGF-binding protein 4 (IGFBP4) is required for bone marrow adipocyte formation. In vitro cultures of primary BMSC exhibit a strong induction of $I g f b p 4$ gene expression with a 15 -fold increase during early stage of adipocyte differentiation [27]. Moreover, primary BMSC from male and female IGFBP4 ${ }^{-1-}$ mice exhibit reduced adipogenesis in vitro [27]. These experiments suggest that IGFBP4 is involved in bone marrow adipocyte formation.

\section{Factors Interacting with Insulin in Regulating BMSC Differentiation Fate to Adipocytes}

\section{Glucose}

Recent studies have demonstrated that the skeleton contributes to systemic nutrient clearance and is the second highest contributing tissue to systemic glucose clearance [3]. A positive relationship between serum glucose and marrow adiposity has been reported [17]. Change in glucose metabolism is an important determinant of marrow fat modulations after gastric bypass surgery and improvement in the glycemic control is associated with reduced marrow fat content [35]. The effect of glucose status on human BMSC adipocyte differentiation has been tested by incubating cells with sera obtained from women with T2D that promote adipocyte differentiation and result in a significant increase in the expression of adipogenic genes (aP2, Lpl, and Ppar $\gamma$ ) and an increase in lipid accumulation [36]. In addition, glycated hemoglobin (HbA1c) is positively correlated with vertebral BMAT content of L1-L3 in diabetic women. Diabetic women with hemoglobin A1C levels $>7 \%$, exhibit higher vertebral BMAT content of L1-L3 compared with patients with $\mathrm{HbA1c}$ levels $\leq 7 \%$ [37]. Moreover, a high level of blood glucose inhibits the proliferation and migration of BMSC and promotes marrow adipocyte formation but not osteoblastogenesis [15]. These studies demonstrate that BMAT formation is affected by glycemic status and glycemic control; however, further studies are needed to determine whether these effects are independent of insulin action.

\section{Receptor for Advanced Glycation End Products}

RAGE is the receptor for advanced glycation end products (AGEs), the products of non-enzymatic glycation and oxidation of proteins that form in hyperglycemic conditions (reviewed in [38]). RAGE signaling pathway plays a role in the pathogenesis of diabetes complications (reviewed in [38]); however, due to its ability to bind multiple ligands and its role in perpetuating and amplifying inflammatory responses, RAGE activation is involved in several inflammatory diseases, such as arteriosclerosis, Alzheimer's disease, arthritis, acute respiratory failure, and sepsis (reviewed in [39]). In T2D model (db/db mice) and T1D model (streptozotocin (STZ)injected mice), the abundance of endogenous BMSC is reduced as determined by colony-forming unit assay [40]. RAGE-KO mice are protected from STZ-induced BMSC reduction and RAGE-KO BMSC exhibit enhanced adipocyte differentiation evidenced by formation of a higher number of Oil Red O-stained mature adipocytes and higher expression 
levels of adipocyte marker genes, e.g., fatty acid-binding protein 4 (Fabp-4), Ppar $\gamma$, and Cebp $\alpha$ compared with wild-type BMSC [40]. Furthermore, RAGE-KO BMSC show a greater potential to differentiate into osteoblasts evidenced by increased expression of osteoblast gene markers, including Alpl, Osx, Bglp, Runx2, Bmp2, and Bmp4 [40]. Based on the above, RAGE signaling may mediate the effect of insulin and glucose in diabetic patients on BMSC properties, and thus, this pathway represents a potential target in the treatment of diabetic bone disease [40].

\section{Parathyroid Hormone}

Diabetic bone disease increases the risk of postmenopausal and age-related osteoporotic fractures [41]. Intermittent parathyroid hormone (PTH) treatment stimulates bone formation and reduces osteoporotic bone loss and osteoporotic fracture risk [41, 42]. Stimulation of type I PTH/PTHrP receptor signaling enhances osteoblast and reduces adipocyte differentiation [42]. In leptin receptor-labeled bone marrow stromal population, PTH treatment shifts cell fate from adipocytic to osteoblastic lineage as evidenced by decreased expression levels of the adipocyte markers such as Cebp $\beta, P p a r \gamma$, and Zfp 467 . Comparably, genetic deletion of the PTH1R in BMSC progenitors resulted in high bone marrow adiposity and low bone mass in rodents [43]. In ovariectomized mice, PTH treatment suppressed the expansion of the BMAT [42]. Therefore, bone marrow adipocytes are responsive to PTH and the positive effects of PTH treatment on bone formation may be mediated by a shift in the differentiation fate of BMSC from adipocytes toward osteoblasts [43]. PTH treatment in mice with T1D increases trabecular bone mass, mineral apposition, and osteoblast surfaces in addition to suppression of osteoblast apoptosis [41], but in contrast to the abovementioned studies, the positive effects of PTH on bone mass are not accompanied by a reduction in diabetes-induced BMAT accumulation [41].

\section{Glucagon-Like Peptide-1}

Glucagon-like peptide-1 (GLP-1) is an intestinal hormone that plays an essential role in the regulation of glucose homeostasis. In human adipose-derived stromal stem cells, GLP-1 stimulated osteoblastic cell differentiation and suppressed adipogenesis [44]. In these cells, inhibiting ERK reversed the anti-adipogenic effect of GLP-1 [44]. In human BMSC, Exendin-4, a stable GLP-1 gut hormone analogue currently used for the treatment of T2D, promotes both adipocytic and osteoblastic differentiations probably due to increased number of committed progenitors. On the other hand, Exendin-4 induces lipolysis in mature adipocytes and does not affect osteoblast metabolic activity [45]. In hindlimb-unloading-induced bone loss rat model, Exendin- 4 treatment enhances bone formation and it decreases adipocyte number in the bone marrow.
Also, Exendin-4 promotes osteoblastic differentiation and inhibits adipocytic differentiation in rat BMSC via regulation PKA/ $\beta$-catenin and PKA/PI3K/AKT/GSK3b signaling [46]. Although GLP-1 may regulate BMSC cell fate and BMAT formation, the effect of GLP-1 on obesity- or diabetesassociated BMAT expansion remains to be determined.

\section{Secretory Factors within Bone Marrow Microenvironment}

Several extracellular factors present within BMSC niche play a role in lineage allocation to adipocytes versus osteoblasts. Our group has identified a number of factors secreted within bone marrow microenvironment that participate in regulation of BMAT formation including DLK1, sFRP-1, and LGMN [12-14] that regulate the differentiation fate of BMSC into osteoblasts versus adipocytes. Among these, DLK1 seems to play a role in regulating insulin effects on the skeleton. DLK1 is co-localized with insulin within the secretory granules of pancreatic $\beta$-cells. Under-caboxylated osteocalcin (GluOCN), a hormone produced by osteoblastic cells, stimulates pancreatic insulin secretion and also production of DLK1. Interestingly, DLK1 antagonizes the effects of insulin on osteoblast production of Glu-OCN and thus represents a mechanism preventing OCN-induced hypoglycemia [47]. These studies demonstrate the close association of secreted factors present in bone marrow and skeleton microenvironment and whole body energy metabolism.

\section{Insulin Signaling in Bone Marrow Adipocytes in Relation to Obesity}

Insulin exerts anabolic effect on the bone metabolism and it has a critical role in the regulation of skeletal development and bone integrity [48]. Insulin signaling represents a key metabolic pathway important for the bioenergetic demand of bone cells [49-53]. Our group has recently examined the effects of obesity on BMAT and its role in regulating skeletal energy demands. We have observed a unique metabolic phenotype of BMAT in obese mice and obese humans that exhibit enhanced insulin signaling, which was opposite to what we observed in peripheral adipose tissue $[18 \bullet \bullet, 22 \bullet \bullet$. In more details, we identified a pro-adipogenic potential of BMSC in mice fed HFD and in obese humans driven by increased insulin signaling associated with enhanced oxidative phosphorylation, leading to BMAT expansion. We have also observed enrichment in a unique BMSC population with high expression of insulin receptor $(\mathrm{IR}+)$ in obese subjects [22・•]. Interestingly, our study suggests that in murine and human obesity, bone marrow microenvironment does not exhibit insulin resistance phenotype. While this seems at variance with previously published data that employed animal models, most 
of the studies have employed insulin receptor (INSR)-deficient mice as a model for insulin resistance $[49,50,51 \bullet \bullet]$. This discrepancy may be explained by the biological differences between INSR-deficient mice, which is a suitable model for insulin deficiency taking place in long-standing T2D and obese subjects who exhibited insulin resistance phenotype but no manifest diabetes. Further, murine studies did not examine the presence of intrinsic changes in BMSC, as the observed insulin resistance may have been related to microenvironmental factors. On the other hand, the study by Wei et al. [51・•] corroborates our findings as it demonstrates that mice with enhanced insulin signaling in bone are protected from the severe systemic insulin resistance phenotype. Our study demonstrates that maintenance of insulin responsiveness in BMSC of obese subjects is "a protective mechanism" allowing fat storage in bone marrow, when peripheral tissues manifest impaired insulin signaling (Table 1 summarizes the major findings of these studies and illustrating similarities and differences between studies). We think that tissue-specific responses to insulin are highly relevant for the bone field with respect to understanding the pathophysiology of obesity and T2D-associated bone disease.

\section{Insulin Signaling and Metabolic Programming of BMSC}

Stem cells existing in states of commitment and differentiation have specific bioenergetic needs that determine their functions $[54,55]$. Bone marrow consists of heterogeneous population of BMSC with different lineage commitments [56, 57]. Our group has recently examined whether metabolic programming of BMSC is upstream of lineage commitment [22] (Tencerova et al., 2019 Bone Research, accepted https://doi.org/10.1038/ s41413-019-0076-5).

Stem cells get energy supply from either glycolysis or oxidative phosphorylation (OxPhos) and they differ in the choice of energy substrate depending on whether they are in the growth or differentiation stage. Pluripotent embryonic stem cells prefer anabolic glycolysis, which is also the preferred metabolic process of rapidly proliferating cells [58, 59]. Hematopoietic progenitor cells exhibit differentiation dependent use of glycolysis or OxPhos [60, 61]. Osteoblast lineage cells employ both oxidative and glycolytic metabolic pathways in undifferentiated state, but during osteoblast differentiation, glycolysis is the preferred energy source [62]. On the other hand, a pre-adipocytic embryonic murine cell line 3T3L1 employs OxPhos during adipocyte differentiation [62]. Thus, commitment to either osteoblasts or adipocytes is associated with a characteristic bioenergetic profile that is maintained during differentiation. We have recently observed that human BMSC and committed murine BMSC progenitors exhibit similar bioenergetic phenotype [22・•] (Tencerova et al., 
Bone Research, accepted https://doi.org/10.1038/s41413-0190076-5). Employing high-throughput technologies including RNAseq and metabolomics, we found that committed murine adipocyte progenitors (named $\mathrm{BMSC}^{\text {adipo }}$ ) and osteoblast progenitors (named $\mathrm{BMSC}^{\text {osteo }}$ ) [57] exhibit a distinct metabolic program that is dependent on insulin signaling with higher oxidative phosphorylation in BMSC ${ }^{\text {adipo }}$ compared with preferable glycolysis in $\mathrm{BMSC}^{\text {osteo }}$. Our findings demonstrate that the BMSC exhibit cellular responses to exogenous metabolic signals, which regulate their differentiation fate and the expansion of osteoblast versus adipocyte progenitor populations dependent on the prevalent metabolic environment. Future studies are needed to investigate the contribution of bioenergetic properties of BMSC to whole body energy homeostasis.

\section{Insulin Signaling and Senescence in Bone Marrow-a Dual Role of this Pathway}

Metabolic pathways play a critical role in aging [63]. Insulin signaling belongs to nutrient sensing pathways that are important not only for energy metabolism but also for regulation of cellular senescence in different cell types [64].

Insulin signaling has been shown to regulate mitochondrial function $[65,66]$, which produces the most of cellular energy in form of ATP but this process is also accompanied with formation of intermediates such as reactive oxygen species (ROS) that play a role as a second messenger. Recent study has demonstrated that ROS increases insulin sensitivity via oxidative modification of the insulin receptor (autophosphorylation) or inactivation of protein tyrosine phosphatases, including PTEN and PTP1B [67]. Thus, this oxidative challenge suggests activation of cellular adaptation via modulation of insulin action and antioxidant system [68-70]. In physiological condition, ROS is also required for adipocyte differentiation, which is controlled by insulin signaling [71]. On the other hand, chronic exposure to ROS in obesity and diabetes is associated with insulin resistance [72] as ROS is also a trigger for cell damage, inflammation, and senescence. Insulin signaling regulates mitochondrial function and biogenesis by inhibiting FOXO1, which tunes redox signaling by maintaining NAD+/NADH ratio for activation of SIRT1/PGC1 $\alpha$ important for normal mitochondrial function [73, 74]. SIRT1 deacetylase activity is essential for prolongation of lifespan and delay of cellular senescence $[75,76]$. Thus, the ratio between mitochondrial ROS production and NAD+/NADH redox complex needs to be maintained at the levels, to which the cellular metabolic capacity can adapt. This regulation can be tissue-specific. Insulin signaling in connection to mitochondrial function has been intensively investigated in liver, muscle, or adipose tissue [65]. However, it has not been studied in this context in BMAT and BMSC. We have recently reported that enhanced insulin signaling in BMSC of obese subjects leads to increased OXPHOS activity accompanied with higher ROS production [22••]. We suggested that the presence of this hypermetabolic status of BMSC leads to accelerated senescence phenotype and consequently impairment of stem cell functions and increased risk of bone fragility in obesity and diabetes (depicted in Fig. 1).

\section{Perspectives}

Obesity and T2D are increasingly recognized as risk factor for bone fractures [77-81]. New therapeutic strategies are needed based on understanding the biological and molecular mechanisms of BMAT formation. Thus, unraveling the relationship between BMAT and bone metabolism at the molecular level is relevant.

Based on our understanding of insulin signaling and BMAT formation, it is possible that inhibiting insulin signaling within BMSC may serve as a protective mechanism against expansion of BMAT. This notion is supported by experience of using TZD in treatment of T2D. These drugs increase insulin sensitivity but led to increased BMAT formation and increased risk for fractures [77, 80, 81].

Other approach is metabolic slowing induced by caloric restriction or nutrient supplementation that can prevent accumulation of unused intermediates from metabolic processes and support mitochondrial functions in metabolically active tissues [82-84]. On the other hand, calorie restriction in animals is associated with increased BMAT formation, but its effects on humans remain to be determined.

Given the interactions among medications and lifestyle modifications/interventions, the relative effect on BMAT metabolic phenotype and insulin signaling within bone microenvironment needs studying to identify specific approaches for prevention and treatment of metabolic bone diseases associated with obesity and T2D.

Author Contributions MT, MO, and MK researched data, wrote the manuscript, and reviewed the final manuscript.

Funding Information This work was supported by a fellowship grant from the Danish Diabetes Academy supported by the Novo Nordisk foundation (MT) and the Novo Nordisk Foundation (MK, NNF15OC0016284) and a research grant from the Odense University Hospital (R29-A1374).

\section{Compliance with Ethical Standards}

Conflict of Interest Michaela Tencerova, Meshail Okla, and Moustapha Kassem declare that they have no conflict of interest.

Human and Animal Rights and Informed Consent This article does not contain any studies with human or animal subjects performed by any of the authors. 
Open Access This article is distributed under the terms of the Creative Commons Attribution 4.0 International License (http:// creativecommons.org/licenses/by/4.0/), which permits unrestricted use, distribution, and reproduction in any medium, provided you give appropriate credit to the original author(s) and the source, provide a link to the Creative Commons license, and indicate if changes were made.

\section{References}

Papers of particular interest, published recently, have been highlighted as:

- Of importance

•- Of major importance

1. Tencerova M, Kassem M. The bone marrow-derived stromal cells: commitment and regulation of adipogenesis. Front Endocrinol (Lausanne). 2016;7:-127.

2. Kim TY, Schafer AL. Diabetes and bone marrow adiposity. Curr Osteoporos Rep. 2016;14(6):337-44.

3. Bartelt A, Koehne T, Todter K, Reimer R, Muller B, BehlerJanbeck F, et al. Quantification of bone fatty acid metabolism and its regulation by adipocyte lipoprotein lipase. Int J Mol Sci. 2017;18(6).

4. Hawkes CP, Mostoufi-Moab S. Fat-bone interaction within the bone marrow milieu: impact on hematopoiesis and systemic energy metabolism. Bone. 2019;119:57-64.

5. Li Q, Wu Y, Kang N. Marrow adipose tissue: its origin, function, and regulation in bone remodeling and regeneration. Stem Cells Int. 2018;2018:7098456.

6. Sebo ZL, Rendina-Ruedy E, Ables GP, Lindskog DM, Rodeheffer MS, Fazeli PK, et al. Bone marrow adiposity: basic and clinical implications. Endocr Rev. 2019;40(5):1187-206.

7. Yue R, Zhou BO, Shimada IS, Zhao Z, Morrison SJ. Leptin receptor promotes adipogenesis and reduces osteogenesis by regulating mesenchymal stromal cells in adult bone marrow. Cell Stem Cell. 2016;18(6):782-96.

8. Holt V, Caplan AI, Haynesworth SE. Identification of a subpopulation of marrow MSC-derived medullary adipocytes that express osteoclast-regulating molecules: marrow adipocytes express osteoclast mediators. PLoS One. 2014;9(10):e108920.

9.• Ambrosi TH, Scialdone A, Graja A, Gohlke S, Jank AM, Bocian C, et al. Adipocyte accumulation in the bone marrow during obesity and aging impairs stem cell-based hematopoietic and bone regeneration. Cell Stem Cell. 2017;20(6):771-84 e6 This study investigated the effect of obesity and aging on the composition and expansion of BMSC subpopulations giving rise to adipocytes, osteoblasts, and chondrocytes using lineage tracing technologies.

10. Pineault KM, Song JY, Kozloff KM, Lucas D, Wellik DM. Hox11 expressing regional skeletal stem cells are progenitors for osteoblasts, chondrocytes and adipocytes throughout life. Nat Commun. 2019;10(1):3168.

11.• Cawthorn WP, Scheller EL, Learman BS, Parlee SD, Simon BR, Mori $\mathrm{H}$, et al. Bone marrow adipose tissue is an endocrine organ that contributes to increased circulating adiponectin during caloric restriction. Cell Metab. 2014;20(2):368-75 This study highlights the importance of bone marrow fat as an active endocrine organ.

12. Jafari A, Qanie D, Andersen TL, Zhang Y, Chen L, Postert B, et al. Legumain regulates differentiation fate of human bone marrow stromal cells and is altered in postmenopausal osteoporosis. Stem Cell Reports. 2017;8(2):373-86.

13. Abdallah BM, Kassem M. New factors controlling the balance between osteoblastogenesis and adipogenesis. Bone. 2012;50(2): $540-5$.

14. Abdallah BM, Jensen CH, Gutierrez G, Leslie RG, Jensen TG, Kassem M. Regulation of human skeletal stem cells differentiation by Dlk1/Pref-1. J Bone Miner Res. 2004;19(5):841-52.

15. Zhu L, Xu Z, Li G, Wang Y, Li X, Shi X, et al. Marrow adiposity as an indicator for insulin resistance in postmenopausal women with newly diagnosed type 2 diabetes - an investigation by chemical shift-encoded water-fat MRI. Eur J Radiol. 2019;113:158-64.

16. Artsi H, Gurt I, El-Haj M, Muller R, Kuhn GA, Ben Shalom G, et al. Sirt1 promotes a thermogenic gene program in bone marrow adipocytes: from mice to (wo)men. Front Endocrinol (Lausanne). 2019;10:126.

17. Li Y, Meng Y, Yu X. The unique metabolic characteristics of bone marrow adipose tissue. Front Endocrinol (Lausanne). 2019;10:69.

18.• Tencerova M, Figeac F, Ditzel N, Taipaleenmaki H, Nielsen TK, Kassem M. High-fat diet-induced obesity promotes expansion of bone marrow adipose tissue and impairs skeletal stem cell functions in mice. J Bone Miner Res. 2018;33(6):1154-65 This study provides an important information about an absence of insulin resistance in bone marrow fat in animal model of diet-induced obesity.

19. Lecka-Czernik B. Marrow fat metabolism is linked to the systemic energy metabolism. Bone. 2012;50(2):534-9.

20. Qiang G, Whang Kong H, Xu S, Pham HA, Parlee SD, Burr AA, et al. Lipodystrophy and severe metabolic dysfunction in mice with adipose tissue-specific insulin receptor ablation. Mol Metab. 2016;5(7):480-90.

21. Irwin R, Lin HV, Motyl KJ, McCabe LR. Normal bone density obtained in the absence of insulin receptor expression in bone. Endocrinology. 2006;147(12):5760-7.

22.• Tencerova M, Frost M, Figeac F, Nielsen TK, Ali D, Lauterlein JL, et al. Obesity-associated hypermetabolism and accelerated senescence of bone marrow stromal stem cells suggest a potential mechanism for bone fragility. Cell Rep. 2019;27(7):2050-62 e6 This clinical study demonstrates that obesity activates insulin signaling in BMSC, which creates senescent microenvironment associated with a higher risk for bone fractures.

23. Yu EW, Greenblatt L, Eajazi A, Torriani M, Bredella MA. Marrow adipose tissue composition in adults with morbid obesity. Bone. 2017;97:38-42.

24. Wang Y, Yang L, Liu X, Hong T, Wang T, Dong A, et al. miR-431 inhibits adipogenic differentiation of human bone marrow-derived mesenchymal stem cells via targeting insulin receptor substance 2 . Stem Cell Res Ther. 2018;9(1):231.

25. Wang N, Li Y, Li Z, Ma J, Wu X, Pan R, et al. IRS-1 targets TAZ to inhibit adipogenesis of rat bone marrow mesenchymal stem cells through PI3K-Akt and MEK-ERK pathways. Eur J Pharmacol. 2019;849:11-21.

26. Tang CY, Man XF, Guo Y, Tang HN, Tang J, Zhou CL, et al. IRS-2 partially compensates for the insulin signal defects in IRS-1(-/-) mice mediated by miR-33. Mol Cells. 2017;40(2):123-32.

27. Maridas DE, DeMambro VE, Le PT, Mohan S, Rosen CJ. IGFBP4 is required for adipogenesis and influences the distribution of adipose depots. Endocrinology. 2017;158(10):3488-500.

28. Dinchuk JE, Cao C, Huang F, Reeves KA, Wang J, Myers F, et al. Insulin receptor (IR) pathway hyperactivity in IGF-IR null cells and suppression of downstream growth signaling using the dual IGFIR/IR inhibitor, BMS-754807. Endocrinology. 2010;151(9):412332.

29. Slaaby R, Schaffer L, Lautrup-Larsen I, Andersen AS, Shaw AC, Mathiasen IS, et al. Hybrid receptors formed by insulin receptor (IR) and insulin-like growth factor I receptor (IGF-IR) have low 
insulin and high IGF-1 affinity irrespective of the IR splice variant. J Biol Chem. 2006;281(36):25869-74.

30. Patel VS, Ete Chan M, Rubin J, Rubin CT. Marrow adiposity and hematopoiesis in aging and obesity: exercise as an intervention. Curr Osteoporos Rep. 2018;16(2):105-15.

31. Ghali O, Al Rassy N, Hardouin P, Chauveau C. Increased bone marrow adiposity in a context of energy deficit: the tip of the iceberg? Front Endocrinol (Lausanne). 2016;7:125.

32. Bredella MA, Torriani M, Ghomi RH, Thomas BJ, Brick DJ, Gerweck AV, et al. Vertebral bone marrow fat is positively associated with visceral fat and inversely associated with IGF-1 in obese women. Obesity (Silver Spring). 2011;19(1):49-53.

33. Menagh PJ, Turner RT, Jump DB, Wong CP, Lowry MB, Yakar S, et al. Growth hormone regulates the balance between bone formation and bone marrow adiposity. J Bone Miner Res. 2010;25(4): 757-68.

34. Fritton JC, Kawashima Y, Mejia W, Courtland HW, Elis S, Sun H, et al. The insulin-like growth factor-1 binding protein acid-labile subunit alters mesenchymal stromal cell fate. J Biol Chem. 2010;285(7):4709-14.

35. Kim TY, Schwartz AV, Li X, Xu K, Black DM, Petrenko DM, et al. Bone marrow fat changes after gastric bypass surgery are associated with loss of bone mass. J Bone Miner Res. 2017;32(11):2239-47.

36. Moseley KF, Doyle ME, Jan De Beur SM. Diabetic serum from older women increases adipogenic differentiation in mesenchymal stem cells. Endocr Res. 2018;43(3):155-65.

37. Baum T, Yap SP, Karampinos DC, Nardo L, Kuo D, Burghardt AJ, et al. Does vertebral bone marrow fat content correlate with abdominal adipose tissue, lumbar spine bone mineral density, and blood biomarkers in women with type 2 diabetes mellitus? J Magn Reson Imaging. 2012;35(1):117-24.

38. Ramasamy R, Yan SF, Schmidt AM. Receptor for AGE (RAGE): signaling mechanisms in the pathogenesis of diabetes and its complications. Ann N Y Acad Sci. 2011;1243:88-102.

39. Chuah YK, Basir R, Talib H, Tie TH, Nordin N. Receptor for advanced glycation end products and its involvement in inflammatory diseases. Int J Inflam. 2013;2013:403460.

40. Aikawa E, Fujita R, Asai M, Kaneda Y, Tamai K. Receptor for advanced glycation end products-mediated signaling impairs the maintenance of bone marrow mesenchymal stromal cells in diabetic model mice. Stem Cells Dev. 2016;25(22):1721-32.

41. Motyl KJ, McCauley LK, McCabe LR. Amelioration of type I diabetes-induced osteoporosis by parathyroid hormone is associated with improved osteoblast survival. J Cell Physiol. 2012;227(4): 1326-34.

42. Yang M, Arai A, Udagawa N, Zhao L, Nishida D, Murakami K, et al. Parathyroid hormone shifts cell fate of a Leptin receptormarked stromal population from adipogenic to osteoblastic lineage. J Bone Miner Res. 2019.

43. Fan Y, Hanai JI, Le PT, Bi R, Maridas D, DeMambro V, et al. Parathyroid hormone directs bone marrow mesenchymal cell fate. Cell Metab. 2017;25(3):661-72.

44. Lee HM, Joo BS, Lee CH, Kim HY, Ock JH, Lee YS. Effect of glucagon-like peptide-1 on the differentiation of adipose-derived stem cells into osteoblasts and adipocytes. J Menopausal Med. 2015;21(2):93-103

45. Luciani P, Fibbi B, Mazzanti B, Deledda C, Ballerini L, Aldinucci A, et al. The effects of Exendin-4 on bone marrow-derived mesenchymal cells. Endocrine. 2018;60(3):423-34.

46. Meng J, Ma X, Wang N, Jia M, Bi L, Wang Y, et al. Activation of GLP-1 receptor promotes bone marrow stromal cell osteogenic differentiation through beta-catenin. Stem Cell Reports. 2016;6(4): 579-91.

47. Abdallah BM, Ditzel N, Laborda J, Karsenty G, Kassem M. DLK1 regulates whole-body glucose metabolism: a negative feedback regulation of the osteocalcin-insulin loop. Diabetes. 2015;64(9): 3069-80.

48. Thrailkill K, Bunn RC, Lumpkin C Jr, Wahl E, Cockrell G, Morris L, et al. Loss of insulin receptor in osteoprogenitor cells impairs structural strength of bone. J Diabetes Res. 2014;2014:703589.

49. Fulzele K, Riddle RC, DiGirolamo DJ, Cao X, Wan C, Chen D, et al. Insulin receptor signaling in osteoblasts regulates postnatal bone acquisition and body composition. Cell. 2010;142(2):309-19.

50. Ferron M, Wei J, Yoshizawa T, Del Fattore A, DePinho RA, Teti A, et al. Insulin signaling in osteoblasts integrates bone remodeling and energy metabolism. Cell. 2010;142(2):296-308.

51.• Wei J, Ferron M, Clarke CJ, Hannun YA, Jiang H, Blaner WS, et al. Bone-specific insulin resistance disrupts whole-body glucose homeostasis via decreased osteocalcin activation. J Clin Invest. 2014;124(4):1-13. This study demonstrates the role of insulin signaling in the skeletal system and regulation of whole body energy metabolism in mice.

52. Kim SP, Li Z, Zoch ML, Frey JL, Bowman CE, Kushwaha P, et al. Fatty acid oxidation by the osteoblast is required for normal bone acquisition in a sex- and diet-dependent manner. JCI Insight. 2017;2(16)

53. Li Z, Frey JL, Wong GW, Faugere MC, Wolfgang MJ, Kim JK, et al. Glucose transporter-4 facilitates insulin-stimulated glucose uptake in osteoblasts. Endocrinology. 2016;157(11):4094-103.

54. Ryall JG, Cliff T, Dalton S, Sartorelli V. Metabolic reprogramming of stem cell epigenetics. Cell Stem Cell. 2015;17(6):651-62.

55. Wellen KE, Thompson CB. A two-way street: reciprocal regulation of metabolism and signalling. Nat Rev Mol Cell Biol. 2012;13(4): $270-6$.

56. Larsen KH, Frederiksen CM, Burns JS, Abdallah BM, Kassem M. Identifying a molecular phenotype for bone marrow stromal cells with in vivo bone-forming capacity. J Bone Miner Res. 2010;25(4): 796-808.

57. Post S, Abdallah BM, Bentzon JF, Kassem M. Demonstration of the presence of independent pre-osteoblastic and pre-adipocytic cell populations in bone marrow-derived mesenchymal stem cells. Bone. 2008;43(1):32-9.

58. Moussaieff A, Rouleau M, Kitsberg D, Cohen M, Levy G, Barasch $\mathrm{D}$, et al. Glycolysis-mediated changes in acetyl-CoA and histone acetylation control the early differentiation of embryonic stem cells. Cell Metab. 2015;21(3):392-402.

59. Hansson J, Rafiee MR, Reiland S, Polo JM, Gehring J, Okawa S, et al. Highly coordinated proteome dynamics during reprogramming of somatic cells to pluripotency. Cell Rep. 2012;2(6):1579-92.

60. Klimmeck D, Hansson J, Raffel S, Vakhrushev SY, Trumpp A, Krijgsveld J. Proteomic cornerstones of hematopoietic stem cell differentiation: distinct signatures of multipotent progenitors and myeloid committed cells. Mol Cell Proteomics. 2012;11(8):286302.

61. Simsek T, Kocabas F, Zheng J, Deberardinis RJ, Mahmoud AI, Olson EN, et al. The distinct metabolic profile of hematopoietic stem cells reflects their location in a hypoxic niche. Cell Stem Cell. 2010;7(3):380-90.

62. Guntur AR, Gerencser AA, Le PT, DeMambro VE, Bornstein SA, Mookerjee SA, et al. Osteoblast-like MC3T3-E1 cells prefer glycolysis for ATP production but adipocyte-like 3T3-L1 cells prefer oxidative phosphorylation. J Bone Miner Res. 2018;33(6):105265 .

63. Barzilai N, Huffman DM, Muzumdar RH, Bartke A. The critical role of metabolic pathways in aging. Diabetes. 2012;61(6):131522.

64. van Heemst D. Insulin, IGF-1 and longevity. Aging Dis. 2010;1(2): 147-57.

65. Cheng Z, Tseng Y, White MF. Insulin signaling meets mitochondria in metabolism. Trends Endocrinol Metab. 2010;21(10):589-98. 
66. Stump CS, Short KR, Bigelow ML, Schimke JM, Nair KS. Effect of insulin on human skeletal muscle mitochondrial ATP production, protein synthesis, and mRNA transcripts. Proc Natl Acad Sci U S A. 2003;100(13):7996-8001.

67. Loh K, Deng H, Fukushima A, Cai X, Boivin B, Galic S, et al. Reactive oxygen species enhance insulin sensitivity. Cell Metab. 2009;10(4):260-72.

68. Ristow M, Zarse K, Oberbach A, Kloting N, Birringer M, Kiehntopf M, et al. Antioxidants prevent health-promoting effects of physical exercise in humans. Proc Natl Acad Sci U S A. 2009;106(21):8665-70.

69. Radak Z, Chung HY, Goto S. Systemic adaptation to oxidative challenge induced by regular exercise. Free Radic Biol Med. 2008;44(2):153-9.

70. Leslie NR, Bennett D, Lindsay YE, Stewart H, Gray A, Downes CP. Redox regulation of PI 3-kinase signalling via inactivation of PTEN. EMBO J. 2003;22(20):5501-10.

71. Tormos KV, Anso E, Hamanaka RB, Eisenbart J, Joseph J, Kalyanaraman B, et al. Mitochondrial complex III ROS regulate adipocyte differentiation. Cell Metab. 2011;14(4):537-44.

72. Qatanani M, Lazar MA. Mechanisms of obesity-associated insulin resistance: many choices on the menu. Genes Dev. 2007;21(12): 1443-55.

73. Lagouge M, Argmann C, Gerhart-Hines Z, Meziane H, Lerin C, Daussin F, et al. Resveratrol improves mitochondrial function and protects against metabolic disease by activating SIRT1 and PGC1alpha. Cell. 2006;127(6):1109-22.

74. Feige JN, Lagouge M, Canto C, Strehle A, Houten SM, Milne JC, et al. Specific SIRT1 activation mimics low energy levels and protects against diet-induced metabolic disorders by enhancing fat oxidation. Cell Metab. 2008;8(5):347-58.

75. Guarente L. Sir2 links chromatin silencing, metabolism, and aging. Genes Dev. 2000;14(9):1021-6.
76. Sasaki T, Maier B, Bartke A, Scrable H. Progressive loss of SIRT1 with cell cycle withdrawal. Aging Cell. 2006;5(5):413-22.

77. Lecka-Czernik B. Bone loss in diabetes: use of antidiabetic thiazolidinediones and secondary osteoporosis. Curr Osteoporos Rep. 2010;8(4):178-84.

78. Lee RH, Sloane R, Pieper C, Lyles KW, Adler RA, Van Houtven C, et al. Glycemic control and insulin treatment alter fracture risk in older men with type 2 diabetes mellitus. J Bone Miner Res. 2019.

79. Vestergaard P. Diabetes and bone fracture: risk factors for old and young. Diabetologia. 2014;57(10):2007-8.

80. Kohler S, Kaspers S, Salsali A, Zeller C, Woerle HJ. Analysis of fractures in patients with type 2 diabetes treated with empagliflozin in pooled data from placebo-controlled trials and a head-to-head study versus glimepiride. Diabetes Care. 2018;41(8):1809-16.

81. Monami M, Cresci B, Colombini A, Pala L, Balzi D, Gori F, et al. Bone fractures and hypoglycemic treatment in type 2 diabetic patients: a case-control study. Diabetes Care. 2008;31(2):199-203.

82. Redman LM, Smith SR, Burton JH, Martin CK, Il'yasova D, Ravussin E. Metabolic slowing and reduced oxidative damage with sustained caloric restriction support the rate of living and oxidative damage theories of aging. Cell Metab 2018;27(4):805-815 e4.

83. Sutton EF, Beyl R, Early KS, Cefalu WT, Ravussin E, Peterson CM. Early time-restricted feeding improves insulin sensitivity, blood pressure, and oxidative stress even without weight loss in men with prediabetes. Cell Metab. 2018;27(6):1212-21 e3.

84. Canto C, Houtkooper RH, Pirinen E, Youn DY, Oosterveer MH, Cen $\mathrm{Y}$, et al. The $\mathrm{NAD}(+)$ precursor nicotinamide riboside enhances oxidative metabolism and protects against high-fat diet-induced obesity. Cell Metab. 2012;15(6):838-47.

Publisher's Note Springer Nature remains neutral with regard to jurisdictional claims in published maps and institutional affiliations. 